Review Article

\title{
Origin, evolution and genome distribution of microsatellites
}

\author{
Eder Jorge Oliveira, Juliano Gomes Pádua, Maria Imaculada Zucchi, Roland Vencovsky \\ and Maria Lúcia Carneiro Vieira \\ Universidade de São Paulo, Escola Superior de Agricultura 'Luiz de Queiroz', Departamento de Genética, \\ Piracicaba, SP, Brasil.
}

\begin{abstract}
Microsatellites, or simple sequence repeats (SSRs), have been the most widely applied class of molecular markers used in genetic studies, with applications in many fields of genetics including genetic conservation, population genetics, molecular breeding, and paternity testing. This range of applications is due to the fact that microsatellite markers are co-dominant and multi-allelic, are highly reproducible, have high-resolution and are based on the polymerase chain reaction (PCR). When first introduced, the development of microsatellite markers was expensive but now new and efficient methods of repetitive sequence isolation have been reported, which have led to reduced costs and microsatellite-technology has been increasingly applied to several species, including non-model organisms. The advent of microsatellite markers revolutionized the use of molecular markers but the development of biometric methods for analyzing microsatellite data has not accompanied the progress in the application of these markers, with more effort being need to obtain information on the evolution of the repetitive sequences, which constitute microsatellites in order to formulate models that fit the characteristics of such markers. Our review describes the genetic nature of microsatellites, the mechanisms and models of mutation that control their evolution and aspects related to their genesis, distribution and transferability between taxa. The implications of the use of microsatellites as a tool for estimating genetic parameters are also discussed.
\end{abstract}

Key words: microsatellites, molecular genetics, genetic structure of populations.

Received: November 23, 2004; Accepted: August 25, 2005.

\section{Introduction}

During the twenty-first century, the protection of biodiversity is expected to be both crucial and continuing, with conservation genetics being of primary importance for avoiding the extinction of most endangered species alongside the ecological, political and economic aspects of biodiversity protection. The application of molecular techniques, including genome approaches, to conservation genetics has made possible the examination of the genetics of species in danger of extinction and genetic analysis has become widely used in conservation research.

Traditional molecular markers have, in general, provided insufficient statistical power and accuracy for estimating genetic differences but the discovery of highly variable loci such as microsatellites means that the statistical power available for determining differentiation between species groups at risk of extinction is now often very high (Hedrick, 2001 and references therein).

Send correspondence to Maria Lúcia Carneiro Vieira, Universidade de São Paulo, Escola Superior de Agricultura "Luiz de Queiroz", Departamento de Genética, Caixa Postal 83, 13400-970 Piracicaba, SP, Brazil. E-mail: mlcvieir@esalq.usp.br.
Microsatellites, also known as simple sequence repeats (SSR) or short tandem repeats (STR), are non-coding repetitive DNA regions composed of small motifs of 1 to 6 nucleotides repeated in tandem, which are widespread in both eukaryotic and prokaryotic genomes (Field and Wills, 1998; Tóth et al., 2000). Broadly used as genetic markers, microsatellites have a particular attribute in that they suffer higher rates of mutation than the rest of the genome (Jarne and Lagoda, 1996). Microsatellites are classified according to the type of repeat sequence as perfect, imperfect, interrupted or composite. In a perfect microsatellite the repeat sequence is not interrupted by any base not belonging to the motif (e.g. TATATATATATATATA) while in an imperfect microsatellite there is a pair of bases between the repeated motifs that does not match the motif sequence (e.g. TATATATACTATATA). In the case of an interrupted microsatellite there is a small sequence within the repeated sequence that does not match the motif sequence (e.g. TATATACGTGTATATATATA) while in a composite microsatellite the sequence contains two adjacent distinctive sequence-repeats (e.g. TATATATATAGTGTGTGT GT). 
In the past few years, microsatellites have attracted the attention of researchers for a number of reasons, including their extensive use in the construction of genetic maps of several types of organisms (Knapik et al., 1998; Cregan et al., 1999), the association between the instability of the number of repeats and human genetic diseases (Mahadevan et al., 1992; Stallings, 1994; O'Donnell and Warren, 2002), their practicability and ease of use in studies of population genetics, and for genotyping and paternity analysis (Wright and Bentzen, 1994; Schlötterer, 2000).

Although originally designed for research in humans, microsatellite analysis has become a powerful tool for research on animals (Schlötterer et al., 1991) and plants (Dayanandan et al., 1997; White and Powell, 1997; Steinkellner et al., 1997; Cipriani et al., 1999; Roa et al., 2000; Collevatti et al., 2001). According to Heywood and Iriondo (2003), microsatellite markers provide relevant information for identifying conservation units and for investigating the genetic processes that take place in populations such as patterns of gene flow, generation of genetic neighborhoods and the incidence of genetic drift. Currently, microsatellite markers are commonly employed for the analysis of plant population genetic structure of both wild (Zucchi et al., 2002) and crop species (Pinto et al., 2003a, b) because of their co-dominant nature and high informativeness.

More recent research based on expressed sequence tags (ESTs) suggest that the frequency of microsatellites in plants is greater than was previously thought, with Morgante et al. (2002) having found that the number of microsatellites per $\mathrm{Mb}$ is about 1844 in Arabidopsis thaliana, 2757 in rice, 2000 in soybean, 1470 in maize and 1796, in wheat.

Until a few years ago, microsatellites were thought to be selectively neutral markers and not affected by selective pressures. However, it is now evident that the expansion of the number of repeats may cause human diseases. For example, Huntington's disease is caused by increases in the length of a CAG motif repeat present in the huntingtin protein gene on human chromosome 4 (Moxon and Wills, 1999), and an increasing number of neurodegenerative disorders have been related to expanded microsatellite repeats, mainly in the tri-nucleotide class (Goldstein and Schlotterer, 1999; Cummings and Zoghbi, 2000; Everett and Wood, 2004). Quite interesting is the fact that microsatellites are preferentially associated with non-repetitive DNA in plant genomes i.e. they frequently occur within and near genes (Morgante et al., 2002).

\section{Genetic Features of Microsatellites}

An homozygous microsatellite locus has the same number of repeats on both homologous chromosomes, whereas a heterozygous microsatellite locus has a different number of repeats for each allele e.g. one allele can contain 9 repeats and the other 10 . However, at the same locus the population as a whole usually contains several alleles each with a different number of repeats, which means that microsatellite markers are very useful for discriminating different individuals. Assuming that $m$ is the number of alleles in a population, the maximum number of different genotypes (NDG) will be $m(m+1) / 2$ and the number of possible heterozygous genotypes (NHG) will be $m(m-1) / 2$, e.g. if $m=48, \mathrm{NDG}=1,176$ and $\mathrm{NHG}=1,128$. The high discriminating power of microsatellites is an important characteristic which justifies their use in population genetic studies and forensic science.

\section{Mutation Mechanisms}

Although microsatellites have been extensively used in a considerable number of studies covering the most varied areas of genetics, the mutational dynamics of these genomic regions is still not well understood (Schlötterer, 2000), although it is known that the mutation rate of microsatellites is much higher than that of other parts of the genome, ranging from $10^{-2}$ to $10^{-6}$ nucleotides per locus per generation (Sia et al., 2000 and references therein).

Several mechanisms have been suggested to explain the high mutation rate of microsatellites, including errors during recombination, unequal crossing-over and polymerase slippage during DNA replication or repair (Strand et al., 1993).

In regard to the inclusion of errors during recombination, Levinson and Gutman (1987) found that strains of Escherichia coli with or without a functional recombination system had a similar mutation rate, suggesting that recombination is not the predominant mechanism in the generation of microsatellite variability.

When unequal crossing-over occurs, there can be drastic changes such as the loss or gain of a large number of repeats. This is because when microsatellite repetitive regions are present, a hairpin (the dark region in Figure 1) can be formed during synapsis, which means that only parts, usually unequal in length, of each chromosome will be exchanged and one chromosome will receive a larger fragment because of the larger number of microsatellite repeats exchanged, the homologues chromosome receiving a smaller number of repeats.

During DNA replication or repair, DNA polymerase slippage can occur in which one DNA strand temporarily dissociates from the other and rapidly rebinds in a different position, leading to base-pairing errors and continued lengthening of the new strand and an increase in the number of repeats (i.e. additions) in the allele if the error occurs on the complementary strand or a decreased number of repeats (i.e. deletions) if the error occurs on the parent strand (Figure 2).

High rates of slippage have been demonstrated but these appear to lead to only small changes in the number of repeats (Hentschel, 1982; Streisinger and Owen, 1985; Schlötterer and Tautz, 1992). Slippage can destabilize microsatellites either because there is no effective repair 


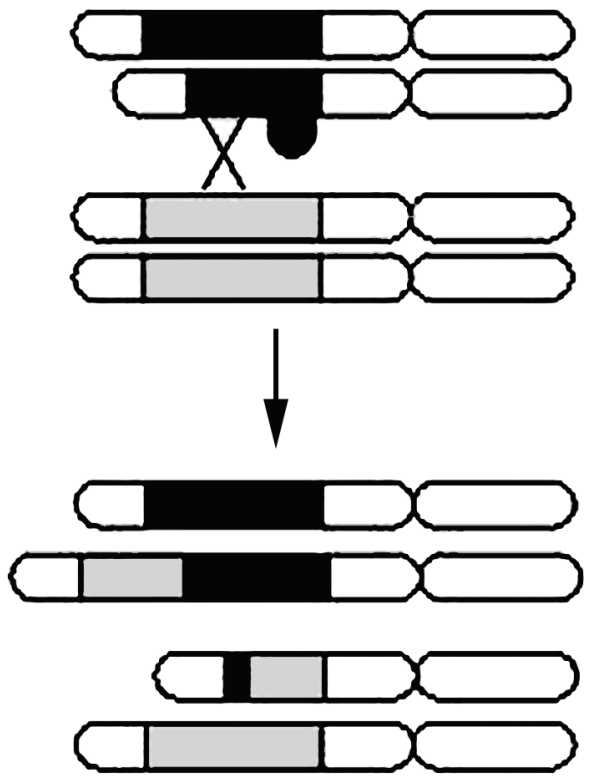

Figure 1 - Unequal crossing-over between homologous chromosomes. Black and gray regions correspond to microsatellite repeat sequences.

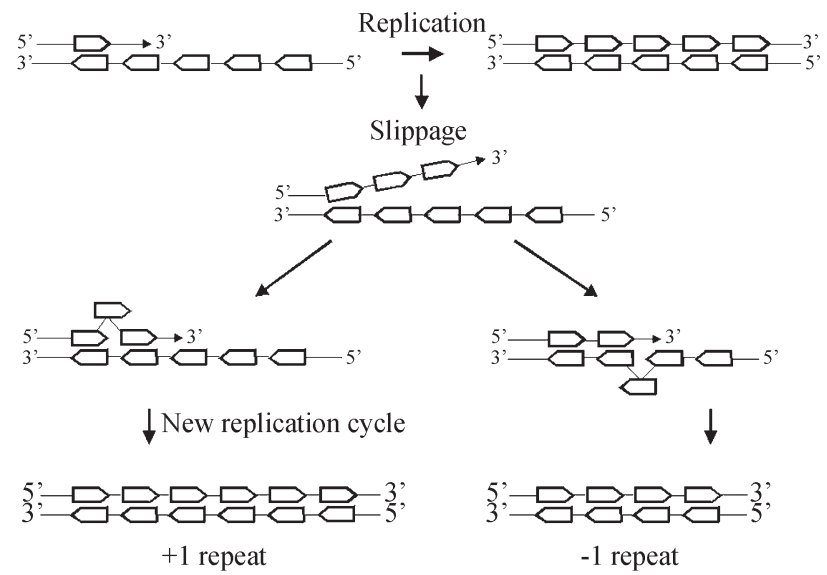

Figure 2 - Slippage during DNA replication. Assume that in the original DNA molecule there were 5 repeats of the motif, symbolized by a box. Slippage leads to the formation of new alleles with 6 and 4 repeats, depending on the strand containing the polymerase error (Modified from Goldsteind and Schlotterer, 1999).

system for DNA loops or because of alterations in DNA polymerase or its cofactors that result in increased slippage rates. Mutations in the genes of the DNA repair system substantially increase (up to 700 times) microsatellite instability in E. coli (Bichara et al., 2000), yeast (Strand et al., 1993; Sia et al., 1997) and mammal cells (Kolodner and Marsischky, 1999) while mutations affecting the DNA polymerase correction domain produce less drastic effects (Sia et al., 2000).

\section{Mutation Rates: The Theoretical Models}

An important question to be answered is which theoretical model should be applied to correctly determine popu- lation genetic parameters obtained from microsatellite data. Mutational models are used to derive the expected number of alleles in a population from the observed heterozygosity and also in the statistical analyses of genetic variation, but all models have some disadvantages when applied to microsatellite data. In general, four models can be used.

\section{Infinite alleles (IA) model}

In this model, each mutation randomly creates a new allele. Applying this model to microsatellite loci, mutations alter the number of repeats. For example, an allele with 10 repeats is considered to be as closely related genetically to an allele with 15 repeats as to one with 16 repeats, i.e. proximity in terms of the number of repeats does not indicate a greater phylogenetic relationship. This is Wright's (1931) classical model in which he uses $F$-statistics.

\section{Stepwise mutation (SM) model}

When a microsatellite locus mutates, it gains or loses a repeat. This implies that two alleles differing by only one motif are more related (i.e. share a more recent common ancestor) than alleles differing by several repeats. Slatkin (1995) proposed a genetic differentiation measure $\left(R_{S T}\right)$ similar to Wright's (1951) $F_{S T}$ and Nei's (1973) $G_{S T}$ but based on the SM model.

The SM model is usually preferred when estimating relations between individuals and population structure, except in the presence of homoplasy (i.e. when two alleles are identical by state but not by descent). Homoplasy may seriously influence population studies involving high mutation rates and large population sizes together with strong allele size constraints (Estoup et al., 2002). The model described by Slatkin (1995) is based on traits with continuous distribution, number of base pairs or number of repeats, and it groups individuals according to the number of repeats.

\section{Two phase (TP) model}

Di Rienzo et al. (1994) introduced this model as an extension of the SM model for studies on microsatellites. It states that most mutational events result in an increase or decrease of one repeat unit, though infrequent alterations of a large number of repeats also occur.

\section{K-alleles (KA) model}

Crow and Kimura (1970) proposed the KA model in 1970 , which assumes that if there are exactly $k$ possible alleles in a given locus then the probability of a given allele mutating into any other is $\mu / \mathrm{k}-1$, where $\mu$ is the mutation rate.

\section{Genesis of Microsatellites}

In yeasts, it seems that no minimum number of repeats is required for microsatellites to evolve (Pupko and Graur, 1999). Rose and Falush (1998) compared the ex- 
pected and observed numbers of microsatellites in the yeast genome and found that long repeats are more common than would be expected by chance and attributed this to slippage. A small number of repeats (fewer than 8 nucleotides, e.g. 2 tetranucleotides, 4 dinucleotides or 8 mononucleotides) is less common than would be expected by chance events, which explains the fact that DNA polymerase slippage is rare.

A study on the origin of microsatellites concluded that a minimum number of repeats (proto-microsatellite) is required before DNA polymerase slippage can extend the number of repeats (Rose and Falush, 1998, Messier et al. 1996). It has been shown that in species that have primates as their common ancestor (e.g. gorillas, chimpanzees and humans) a GA mutation at the $\eta$-globin locus changed the sequence ATGTGTGT to ATGTATGT, thus creating a microsatellite (ATGT) $)_{2}$ which evolved into (ATGT) $)_{4}$ in African monkeys and (ATGT) $)_{5}$ in humans (Messier et al. 1996).

Zhu et al. (2000) conducted an elegant study on mutated human genes and demonstrated that more than $70 \%$ of all 2 to 4 nucleotide insertions resulted in 2 to 5 new repeats, most of which are not extensions of pre-existing repeats but new microsatellites originating from random sequences. This indicates that the types and processes that lead to the expansion of microsatellite loci and polymorphism also occur with few repeats.

In humans, as compared to yeasts, a completely different mechanism for generating microsatellites has been deduced from the association of microsatellites with retrotransposons (Nadir et al., 1996). The authors speculated that microsatellites rich in A-base were generated by the extension of terminal 3' of retrotranscripts, similarly to the mRNA polyadenylation mechanism.

According to Arcot et al. (1995), the Alu SINEs (interspersed nuclear elements) family is largely dispersed in the primate genome, and is likely to contribute to the genesis of microsatellites due to the presence of adenine-rich regions at the 3' terminal and within the sequence. The association between microsatellites and $A l u$ elements can be explained in terms of three mechanisms: 1) the Alu element integrates into a pre-existing microsatellite, resulting in repeats of the microsatellite flanking the element; 2) Alu elements are integrated with mutations that are introduced in the primary transcript during reverse transcription, with the mutation acting as a nucleus for microsatellite genesis; and 3) the accumulation of random mutations in the poly(A) tail of Alu elements, followed by the expansion of this region by slippage or intra-allelic recombination to produce microsatellites. Mechanism 1 assumes that microsatellites are present a priori to the insertion of Alu elements, whereas mechanisms 2 and 3 are based on indirect evidence suggesting that the internal adenine-rich region and oligo(dA) 3' terminal of the Alu elements are sources for microsatellite genesis.
While such an association has been found to apply to a great number of organisms, a high density of transposable elements does not always coincide with a high density of microsatellites (Lin et al., 1999). Therefore, retrotransposition as a generalized mechanism for microsatellite genesis remains questionable.

Ramsay et al. (1999) analyzed microsatellite flanking sequences in hops and showed that a high proportion of clones were homologous to known transposons. An association was found between the repetitive dispersed element R173 and the transposons BARE-1, WIS2-1A and PREM1. The microsatellites found in Ramsey's study were of two types, those with single sequences in the flanking region and those associated with retrotransposons and other repetitive dispersed elements. Three subtypes compose the second type: a) those positioned at terminal 3 ' of the transposon with a single sequence at the other terminal; b) those positioned at terminal 5'; and c) those in which the internal sequence of the transposon is homologous in both flanking regions.

\section{Microsatellite Size Distribution in Genomic Sequences}

The number of repeats is a crucial factor determining the evolutionary dynamics of microsatellite DNA, and it is important to investigate which parameters influence the length of repeats. Taking the simplest model of microsatellite evolution, DNA slippage is a symmetrical process and, consequently, the number of repeats added is on average the same as the number removed.

Kruglyak et al. (1998) proposed a model for the size distribution of microsatellites in genomic sequences that does not assume selection or mutation to be size-related processes, infinite growth being prevented by the accumulation of base substitutions at microsatellite loci. An important aspect of this model is that it assumes a constant base substitution rate in which the slippage rate can be determined on the basis of the microsatellite length distribution in genomic sequences. This means that species with short microsatellites (e.g. Drosophila melanogaster) should have lower microsatellite mutation rates than species with longer microsatellites.

We can test this theory by comparing the mutation rates of microsatellites with equal number of repeats. Given that microsatellite loci are quite well conserved in different species, it is possible to determine whether the number of repeats diverges according to species. A comparison of microsatellites from chimpanzees and humans showed that human microsatellites contain many more repeats (Amos et al., 1996; Cooper et al., 1998).

\section{Genome Distribution}

Microsatellites are not regularly distributed within a single genome due to differences in their frequencies 
within coding and non-coding sequences (Arcot et al., 1995; Wilder and Hollocher, 2001) and the possible functional roles of different repeats (Valle, 1993). The frequency of genomic microsatellites also varies per taxon, in terms of absolute numbers of microsatellite loci and preferential repeats (Hancock, 1999). In plants, the estimated frequency is $0.85 \%$ in Arabidopsis and $0.37 \%$ in Zea mays while in fish it is $3.21 \%$ in Tetraodon nigroviridis and $2.12 \%$ in Fugu rubripes (Crollius et al., 2000) and in Homo sapiens chromosome 22 the microsatellite frequency is $1.07 \%$ whereas in the whole Caernorhabditis elegans genome it is only $0.21 \%$ (Tóth et al., 2000).

According to Morgante et al. (2002), microsatellite frequency differs amongst some plant species i.e. Arabidopsis, maize, soybean, wheat and rice, and is high in Arabidopsis and lower in species with comparatively larger genomes such as maize and wheat. Morgante et al. (2002) point out that there is a significant positive linear relationship between microsatellite frequencies and the percentage of single copy DNA, suggesting that microsatellites should be more frequent within single copy DNA than repetitive DNA. The suggestion that microsatellite frequency is a function of the relative proportion of single copy DNA rather than the size of the genome as a whole is interesting, although this contradicts studies affirming that microsatellites are elements derived from repetitive sequences and that an increase in microsatellite density is closely-related to an increase in genome size (Schlötterer and Harr, 2000).

Due to the high microsatellite mutation rate it is to be expected that coding regions have a low microsatellite density because if they do not these regions would be significantly altered, possibly leading to loss of functionality. Comparative studies (Tóth et al., 2000) in both coding and non-coding regions of different species have confirmed this hypothesis by showing that only tri- and hexa-nucleotides are to be found in excessive numbers over a wide range of repeat unit sizes. In contrast, other types of repeats were much less frequent in coding regions than in non-coding regions. This means that selection against mutations that change the reading frame of a gene restrict the presence of microsatellites in coding regions, while microsatellites with repeats in multiples of three develop evenly in both regions (Metzgar et al., 2000). Obviously, this is related to the fact that RNA bases are read as triplets.

The density of perfect and imperfect microsatellites in genomic regions and expressed sequence tags (ESTs) of Arabidopsis thaliana, Oryza sativa, Glycine max, Zea mays and Triticum aestivum has been assessed by Metzgar et al. (2000) and confirmed by Morgante et al. (2002), both of whom showed that different selective pressures seem to be acting on 5' and 3' untranslated regions (UTRs) and open reading frames (ORFs) of transcription units. These authors found that microsatellite frequency at the 3' UTR region is higher than that expected for the whole genome, with triand tetra-nucleotides contributing markedly to this in- crease. Moreover, the 5' UTR region shows a much higher microsatellite frequency than other genomic fractions, and this is due to the presence of di- and tri-nucleotides, principally AG/CT and AAG/CTT repeats. The difference in selective pressure between the $3^{\prime}$ and 5' UTR regions is clearly due to the higher frequency of CT and CTT repeats

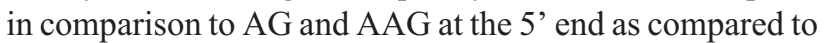
the 3' end.

The contrasting frequency data for different genomes strongly suggests that microsatellite distribution is not merely a reflection of the base composition of the genome but that the DNA repair system plays an important role in determining microsatellite distribution in different species.

Tóth et al. (2000) reported that the total number of 1 to 6 repeat microsatellites varies depending on the taxonomic group concerned, ranging from 13,889 (approximately 429 per $\mathrm{Mb}$, excluding single-base repeats) in Rodentia, to 4,139 (154 per Mb) in Embryophyta, 3,004 (99 per Mb) in Sacharomyces cerevisiae and 2,139 (88 per Mb) in Caernorhabditis elegans. Since $1 \mathrm{Mb}$ corresponds to 2,000 non-overlapped clones with insert sizes of approximately $500 \mathrm{bp}, 21.45 \%$ positive clones in rodents and $4.4 \%$ in C. elegans would be expected using traditional methods for isolating microsatellites. However, when specific repeats are focused, the expected frequency of any tri- or tetra-nucleotide repeat is less than $1 \%$ of positive clones in all taxa. Song et al. (2002) analyzed $4.5 \mathrm{Mb}$ of the wheat genome and estimated that the occurrence of tri-nucleotides with eight or more repeats was $3.0 \times 10^{4}$ for (TAA/ATT) $2.3 \times 10^{4}$ for $(\mathrm{CTT} / \mathrm{GAA})_{\mathrm{n}}, 1.2 \times 10^{4}$ for $(\mathrm{CAA} / \mathrm{GTT})_{\mathrm{n}}, 2.3 \times$ $10^{3}$ for $(\mathrm{CAT} / \mathrm{GTA})_{\mathrm{n}}$ and $1.5 \times 10^{3}$ for $(\mathrm{GGA} / \mathrm{CCT})_{\mathrm{n}}$.

Lin et al. (1999) showed that there was a strong reduction in the density of di-nucleotide microsatellites around the centromere of chromosome 2 of A. thaliana. This tendency was also found in Drosophila (Pardue et al., 1987; Lowenhaupt et al., 1989). Interestingly, the underrepresentation of microsatellites in these genomic regions with a high density of transposons contrasts with the association between microsatellites and the 3' region of retrotransposons of humans (Nadir et al., 1996). If a causal correlation exists between microsatellite genesis and transposon insertion, a higher microsatellite density would be expected in the centromere region.

Non-random microsatellite distribution can also be detected on a more refined scale. Microsatellites that tend to form clusters, leading to non-random distribution in sequences smaller than $15 \mathrm{~kb}$ (Bachtrog et al., 1999), being found in D. melanogaster. Similarly, microsatellite cloning frequently reveals more than one microsatellite sequence in a clone and also indicates that the microsatellites are organized in clusters (Estoup et al., 1999).

\section{Functional Importance of Microsatellites}

Microsatellites can have either a neutral effect on the genome or perform important functions in particular spe- 
cies. Some reports indicate that microsatellites are associated with the regulation and/or functioning of genes, for example $(\mathrm{CT})_{\mathrm{n}}$ motif microsatellites at the 5' UTR region of certain Arabidopsis genes play a role in anti-sense transcription (Kashi and Soller, 1999 and references therein).

Microsatellites are known to be related to pathogenicity and genomic variability in microorganisms and many examples of microsatellites associated with the modulation of microbial gene expression have been identified (Jackson et al., 1997; Field and Wills, 1998; Saunders et al., 1998). For instance, tetra-nucleotide repeats are present within the ORFs in genes coding for Haemophilus influenzae lipopolysaccharides, with variation in repeat number influencing protein production (Belkum, 1999). Repetitive microsatellite-like sequences have also been found in a number of virulence genes in pathogens (Hood et al., 1996).

The Adaptive Peaks Theory (Wright, 1931; 1932) and the fact that the frequency of a microsatellite allele represents a maximum local adaptive value for the population suggests that the majority of mutations generating new alleles result in gene variants of lower local adaptive value.

A number of authors have suggested another function for microsatellites and show that di-nucleotide repeats can act as recombination hot spots (Treco and Arnheim, 1986; Wahls et al., 1990; Bailey et al., 1998). This microsatellite function allows populations to recover genetic variation lost through genetic drift and rapidly adjust to evolutionary demands (Foster and Trimarchi, 1994; Rosenberg et al., 1994).

There is strong evidence that microsatellites can be found upstream of the promoter region and thus regulate the expression of eukaryote genes. For instance, the regulation of several genes depends on the binding of GAGA transcription factors to a small segment of the microsatellite composed of CT repeats present at the first intron promoter of these genes (Biggin and Tjian, 1988; Gilmour et al., 1989), GAGA binding appearing to activate transcription by removing nucleosomes from the promoter or separating the gene from the position effect (O'Donnell et al., 1994).

\section{Microsatellite Transferability}

Progress in the use of microsatellites has encountered setbacks due to the high cost of developing specific primers. However, many studies have shown that primer pairs designed for one species can be used for other species of the same genus (Isagi and Suhandono, 1997; Cipriani et al., 1999) or even for different genera of the same family (White and Powell, 1997; Roa et al., 2000; Zucchi et al., 2002), this microsatellite attribute being known as transferability or cross-species amplification.

Transferability can be a very important factor in facilitating the use of microsatellites because it reduces costs when working on taxa with low microsatellite frequencies or from which microsatellites are difficult to isolate. Micro- satellite transferability amongst related species is allowed by the homologous nature of the DNA sequence in microsatellite flanking regions. However, as expected, the successful amplification rate declines as genetic divergence between species increases (Primmer and Merilä, 2002).

It is worth noting that studies on both humans (Rubinsztein et al.; 1995; Morin et al., 1998) and birds (Ellegren et al., 1995) have shown that the degree of microsatellite polymorphism is not transferable, i.e. high levels of polymorphism detected in one species may not be found at the correspondent locus of another species after primers have been transferred.

In plants, conserved microsatellite loci have been observed across cultivars, subspecies and related species (Métais et al., 2002). Zucchi et al. (2003) were successful in transferring primers originally developed for Eucalyptus spp. (Brondani et al., 1998) to Eugenia dysenterica, both of which are members of the same family but separated by a considerable phylogenetic distance. In this case, $3 \%$ microsatellite locus amplification was possible but about $30 \%$ of the primers amplified non-specific PCR products, revealing the occurrence of mutational events in the primerbinding region.

Working with birds, Lillandt et al. (2002) were successful in using primers originally developed for 18 Corvidae species in Perisoreus infaustus, although some primers that did not produce good quality amplified products had to be redesigned in order to amplify the original locus. This supports the hypothesis that transferability is not overly dependent on phylogenetic proximity. Microsatellite transferability is very advantageous when dealing with birds because there is a low frequency of microsatellites in avian genomes.

In felines, 18 primers developed for Panthera tigris sumatrae showed total transferability to 11 species belonging to three other feline genera, Felis, Acinonyx and Neofelis was also demonstrated (Williamson et al., 2002).

However, very low levels of transferability have been reported in the amphibian genera Triturus (Garner et al., 2003) and Rana (Primmer and Merillä, 2002), possibly due to the fact that amphibians have a very large genome, twice as big as mammals and four times that of birds. These two studies not only show that phylogenetic proximity is a predominant factor in successful transfer but also that transferability is probably affected by other factors such as the size and complexity of the genome concerned and whether or not the microsatellite belongs to a coding region.

\section{Plant Population Structure: The Genetic Power of Microsatellites}

Compared with other classes of markers microsatellites are highly polymorphic, because of which they have been used not only to answer several questions related to plant population genetics, such as gene flow and paternity 
analysis (Wright and Bentzen, 1994), but also for the study of natural plant populations (Collevatti et al., 1999; Daynandan et al., 1997).

Knowledge of the distribution of genetic variability between and within natural plant populations is essential to adopt competent strategies for ex situ and in situ germplasm conservation and microsatellites are extremely useful for estimating genetic population parameters as (i) population structure, (ii) parentage and paternity analysis and (iii) gene flow, all of which will be discussed in more detail below.

\section{Genetic structure of populations}

The most efficient measure to assess population structure is based on Wright's $F$-statistics (1951), Wright's inbreeding coefficient $\left(F_{S T}\right.$, also called $\theta$ ) being particularly useful for analysing microsatellite markers because it is able to discriminate between alleles, especially that rare ones, although $F_{S T}$ produced using such markers can sometimes be overestimates of the true value.

Microsatellite markers include loci with a large number of alleles, but one question that should be asked is whether a large number of loci or a large number of alleles is more important in genetic assessment. Working on the relationship between the allele number and the coefficient of variation of four genetic distances, Kalinowski (2002) used simulated data to show that highly polymorphic loci provided better estimates of genetic distance than less polymorphic loci and that increased allele number was associated with a decrease in the coefficient of variation of each of the four genetic distances studied. These results show that there is no requirement to examine either highly polymorphic loci or large numbers of loci, the only requirement being that a sufficient number of alleles is examined.

However, the high mutation rate of microsatellites can also invalidate many assumptions used in some conventional population structure analysis because different populations may share homoplasic alleles at frequencies that depend on both the rate and the details of the mutation process (Estoup et al., 2002). When such effects are ignored the rate of gene flow or genetic introgression can be overestimated (Balloux et al. 2000). Slatkin (1995) developed the $R_{S T}$ statistic (also called, analogous to $F_{S T}$ ) to take into account the effects of mutation, but although $R_{S T}$ performs better than $F_{S T}$ in some circumstances it can also be sensitive to details of the mutation process (Balloux and Goudet, 2002).

Since mutation rate varies widely between loci within species (Di Rienzo et al. 1998) one advantage of loci with a high mutation rate is that genetic differentiation reaches equilibrium faster, offering the possibility of obtaining estimates from larger and more widely spaced populations. Using a microsatellite data set from Mauritian skinks, Nichols and Freeman (2004) proposed a method for analyzing genetic data to obtain separate estimates of population size and migration rate for sampled populations without precise prior knowledge of mutation rates at each locus.

When working with microsatellites and low migration rates, the $F$-statistic is sensitive to the mutation rate but, unlike the situation under a strict stepwise mutation model, under these conditions $R_{S T}$ is independent of the mutation rate and, due to its high associated variance, can be less accurate at reflecting population differentiation than $F_{S T}$ (Balloux and Lugon-Moulin, 2002). Moreover, $R_{S T}$ will be deflated when the mutation pattern includes mutations involving more than one repeat when the number of possible allelic states is finite (Slatkin, 1995).

The estimation and comparison of both $F$ and $R$-statistic is especially relevant for critical comparison and careful interpretation of data and may give the most valuable information about the genetic structure of a population. Collevatti et al. (2001) used microsatellite loci to investigate the population genetic structure of the endangered tropical tree Caryocar brasiliense and found that $F_{S T}$ was significant lower (0.07) than $R_{S T}(0.29)$ over all loci. This was due to the high and variable mutation rates of microsatellites that usually display high levels of within-population heterozygosity. Slatkin (1995) states that statistics such as $F_{S T}$, which are based on an infinite allele model and consider alleles to be identical by descent, tend to underestimate population differentiation and produce lower values than their corresponding $R_{S T}$ values. In some cases, however, no significant differences have been found between $F_{S T}$ and $R_{S T}$ values, examples being the assessment of genetic structure in populations of Mesoamerican big-leaf mahogany (Swietenia macrophylla, Meliaceae) carried out by Novick et al. (2003), which produced similar overall $F_{S T}$ $(0.109)$ and $R_{S T}(0.177)$ values, and the study of mahogany (S. macrophylla) by Lemes et al. (2003) in which the overall values of $F_{S T}(0.097)$ and $R_{S T}(0.147)$ were again quite close, a further example being the study of Bowen et al. (2005) with loggerhead turtle (Caretta caretta) that again produced similar $F_{S T}(0.002)$ and $R_{S T}(<0.001)$ values.

Another important point regarding the use of microsatellites for genetic analysis of populations has been raised by Petit et al. (2005) who suggested that microsatellite loci with more repeats generally show higher mutation rates (probably because DNA slippage increases in proportion to the number of repeats). In addition, if genetic diversity depends on mutation rate and mutation rate itself depends on the number of repeats subsequently there should be a relationship between microsatellite genetic diversity and the mean number of repeats (MNR). Petit et al. (2005) proposed using allele size and the polymorphism rate of chloroplast microsatellite loci to standardize the level of diversity when microsatellites differ in size and investigated the relationship between the MNR and genetic variation as a prerequisite to comparative studies of genetic diversity. Their findings suggested that the greater allelic richness 
found in some species remains significant after controlling for the number of repeats.

\section{Parentage and paternity analysis}

Plant paternity analysis and gene flow studies have often employed microsatellite markers because unlike allozyme loci, which do not have sufficient variability to determine parentage by exclusion (Chakraborty et al., 1988), each microsatellite locus has many relatively rare alleles and in most case an individual can be excluded from paternity using only a few loci (Dow and Ashley, 1996; Dow et al. 1995).

Chase et al. (1996) used four microsatellite loci and six allozyme loci to estimate paternity exclusion in Pithecellobium (Mimosoideae) and found that not only were microsatellite loci powerful tools for the analysis of population structures but also provide a means for accurately examining both gene flow and paternity, two important parameters in conservation biology.

Concerning relationship coefficients, a problem arose when the term coefficient de parenté (proposed by Malécot, 1948) was translated as coefficient of relationship ( $f$ ) that had already been used by Wright (1922). Thus coefficient de parenté is variously known as kinship (Malécot, 1948), parentage (Kempthorne, 1957) and coancestry (Falconer, 1960).

Kinship is usually calculated either by genetic methods, which employ molecular markers to estimate relatedness based on a quantitative measure of kinship or by genealogical methods that employ qualitative pedigree data based on relationships such as full sibs, half sibs, father and son, etc.

Bernardo et al. (1996) used relationship coefficients to construct a mean genetic relation matrix for use with a best linear unbiased prediction (BLUP) model to calculate combination capabilities and additive and dominant genetic values. Using this methodology it is possible to select genotypes controlling the relationship level (an inverse measure associated with the effective population size; Souza and Sorrels, 1989) and to specify the minimum genetic distance for varietal protection (Hunter, 1989) in light of the fact that when a population is submitted to strong selection variability can be lost.

Molecular markers were not widely available until the 1980s, before which relationship coefficients were estimated using pedigree data but this type of data suffers from the disadvantage that it requires large amounts of historical information that is rare for plants and generally unavailable for natural populations.

Allozymes are not the best markers for estimation relationship because of their restricted ability for sampling the genome as a whole, the most effective marker for this type of estimation being microsatellites as they are codominant and hypervariable (and therefore able to distinguish between closely-related individuals), are abundant in sev- eral genomes and are generally used in conjunction with the PCR. The fact that microsatellite studies employ PCR is the main reason why geneticists in general prefer microsatellite markers as opposed to restriction fragment length polymorphic (RFLP) markers, which although codominant are not PCR-based. In general, only 30-40 microsatellite loci are needed to provide a satisfactory estimate of relationship (Blouin, 2003).

The reason why it is best to use codominant markers to estimate relationship coefficients is the need to discriminate between alleles since, in heterozygous diploids, once we know two alleles at a specific locus it is possible to calculate its complete allelic and genotypic composition. Such considerations indicate that microsatellite markers are the most informative marker for calculating relationship coefficients. Several papers discussing how relationship coefficients can be produced using molecular markers have been published (Queller and Goodnight, 1989; Li et al., 1993; Lynch and Ritland, 1999; Wang, 2002), all of which have concluded that a large number of markers and individuals must be used and that this is particularly important when maximum likelihood estimators are employed (Ritland, 1996). A good example of the use of a large number of microsatellite markers is the study of Bowers et al. (1999) who used 32 microsatellites loci to detect the relationships between 300 grape cultivars, the results showing that most cultivars originated from only a single pair of Pinot and Gouais blanc parents that were widespread in northeastern France during the middle ages.

Another important point is that the markers used for calculating relationships must be independent because if they are not the precision of the estimates will be low (Thompson and Meagher, 1998), this is the reason why all relationship models need to incorporate data from independent loci. Since microsatellites are able to distinguish between alleles, they are the most powerful molecular tool for relationship analyses such as paternity testing that require a high level of precision. This type of analysis has a fundamental role in plant genetics, because it can provide the information necessary to detect the parent of a specific individual in a population. To exclude a random individual from paternity, paternity analysis uses exclusion-probability techniques (Weir, 1996) which depend on the allele frequencies for that locus but not on the genotypes.

Due to its forensic importance, much paternity testing research has been carried out on humans but is equally applicable to plants. In human paternity testing, the conditional probability that a specific man is not the father given the joint probabilities of mother-child combinations is given by the following equation:

$$
Q=\sum_{u} P_{u}\left(1-P_{u}\right)^{2}-\frac{1}{2} \sum_{U} \sum_{V \neq U} P_{U}^{2} P_{V}^{2}\left(4-3 P_{U}-3 P_{V}\right)
$$

where $P$ is the allelic frequency, $U$ is the $u$-th allele and $V$ the $v$-th allele and $Q$ is the overall probability of exclusion. 
It is easy to understand that as more alleles are identified the importance of a particular locus increases, analogous to the increase in exclusion probabilities as the number of loci used is increased. When several independent loci are involved and $Q_{l}$ is the exclusion probability for locus $l$ the overall probability of exclusion $(Q)$ is given by Weir (1996) as:

$$
Q=1-\prod_{l}\left(1-Q_{l}\right)
$$

As recommended by the Combined DNA Index System (CODIS), human paternity tests use 13 microsatellite loci to give a $Q$ value of $1 \times 10^{-4}$ (Chakraborty et al., 1999) but if less loci are used then the $Q$ value will be higher (i.e. more towards 1 , indicating a lower value of exclusion), with, for example, two microsatellite loci with 10 alleles of equal frequency will give a $Q$ value of 0.96 .

\section{Gene flow}

As pointed out by Avise (1994), loss of genetic variability is the central topic in conservation genetics because small populations (especially of allogamous species) occurring in fragmented areas can suffer from inbreeding depression leading to the loss of heterozygosity, genetic diversity and adaptivity.

Gene flow is fundamental for the maintenance of metapopulations because it allows genetic diversity to be maintained by acting directly on the population structure and against random genetic drift. Thus gene flow results in homogenization of allelic frequencies and exactly the opposite effect to genetic drift which tends to make populations genetically more heterogeneous. Gene flow can be quantified indirectly using $F_{S T}$ estimates, the number of private alleles, space autocorrelation and coalescence or directly using morphological markers and paternity analysis. In plants, paternity analysis is the most widely-used method for estimating direct gene flow and by analyzing several loci estimates can be made of the probability of an individual plant being the most probable male parent of a specific offspring among all possible male plants in a particular population. Once the male parent is identified, the pattern of pollen movement can be determined, although the applicability of this methodology is limited to small populations.

In population genetics, the most usual procedure used to quantify gene flow between populations is based on
Wright's infinite-alleles model (see Slatkin, 1995), which assumes migration-drift equilibrium among all populations. Estimates of gene flow based on the analysis of genetic structure of populations can be obtained using the $F_{S T}$ statistic. Gene flow estimated by this method is known as apparent gene flow because it assumes that the genetic structure of the population fits an island model in which there is equilibrium between migration and genetic drift. Under this assumption $F_{S T}$ is a function of the number of migrants per generation, $N m$, where $N$ is the population size and $m$ is the proportion of migrants per generation, the relationship between $F_{S T}$ and $N m$ being:

$$
N m=\frac{1}{4}\left(\frac{1}{F_{S T}}-1\right)
$$

Estimated $\mathrm{Nm}$ values for tropical species are generally higher than 1.0 (Ciampi, 1999; Lemes et al., 2003), with Wright (1951) stating that when $N m$ is higher than 1.0 or when there is one or more individual migrant per generation the effect of migration is sufficient to oppose the drift effect. This simple method for estimating gene flow has been used widely in conservation studies. The estimated gene flow based on $F_{S T}$ for some tropical species is given in Table 1 where it can be seen that the values ranged from 0.75 to 5 , although special care should be taken in interpreting these estimates because, as previously stated, gene flow estimates based on $F_{S T}$ may not be reliable. However, it is interesting to note that $E$. dysenterica population showed the lowest gene flow ( $\mathrm{Nm}=0.75$ migrants per generation) and it is probably in serious risk, while for C. langsdorf the situation is less drastic because the estimated flow of migrants was 5 per generation.

Gaggiotti et al. (1999) conducted simulation studies in which they compared two procedures for estimating gene flow $(\mathrm{Nm})$ based on microsatellite data. These authors compared $N m$ values obtained using Wright's $F_{S T}$ statistic which is defined on the basis of the variance of gene frequencies with $R_{S T}$ values (Slatkin, 1995) which is estimate from the variance of the length of the allele, the underlying genetic model assuming stepwise mutations and constraints in the range of allelic size classes. The results of these simulations suggested that the use of microsatellite loci can lead to serious overestimation of $\mathrm{Nm}$ especially when population sizes are large $(N>5,000)$ and the range of constraints

Table 1 - Microsatellite $F_{S T}, R_{S T}$ and gene flow estimates calculated for populations of some tropical species. Note that $N m_{\mathrm{A}}$ was based on $F_{S T}$ while $N m_{\mathrm{B}}$ was calculated from $R_{S T}$.

\begin{tabular}{lccccl}
\hline Species & $F_{S T}$ & $N m_{A}$ & $R_{S T}$ & $N m_{B}$ & Reference \\
\hline Swietenia macrophylla & 0.097 & 2.327 & 0.147 & 1.450 & Lemes et al. (2003) \\
Caryocar brasilliense & 0.070 & 3.572 & 0.290 & 0.612 & Collevatti et al. (2001) \\
Copaifera langsdorfi & 0.050 & 5.00 & 0.031 & 7.810 & Ciampi (1999) \\
Anopheles arabiensis & 0.069 & 3.372 & 0.025 & 9.750 & Donnelly et al. (2004) \\
Eugenia dysenterica & 0.250 & 0.750 & 0.267 & 0.687 & Zucchi et al. (2003) \\
\hline
\end{tabular}


are high. For large population sample sizes $\left(n_{s}=50\right)$ when many microsatellite loci $\left(n_{l}=20\right)$ were present $R_{S T}$ performed better than $F_{S T}$ while when sample sizes were moderate or small $\left(n_{s}=10\right)$ and the number of loci was low $\left(n_{l}=20\right) F_{S T}$ performed better than $R_{S T}$ in estimating $\mathrm{Nm}$.

These results highlight the fact that when microsatellites are used in interpopulation diversity and gene flow studies of natural populations there is no standard biometric estimation procedure adequate for all situations and procedures should be chosen according the characteristics of the data.

\section{Effective population size}

Gene diversity or expected heterozygosis $(h)$ (Weir, 1996) is an important parameter in studies on the genetic structure of populations. At an intrapopulation level $h$ is defined on a locus basis as being $h=1-\sum p_{u}^{2}$, where $p_{u}$ is the frequency of the $u^{\text {th }}$ allele at that locus. For estimation, an average value is generally obtained. It can be shown that the expression cited above can also be written as $h=1-1 / m-m \sigma_{p}^{2}$, for a locus with $m$ alleles where $\sigma_{p}^{2}$ is the variance of the allelic frequencies of the locus. The $h$ parameter is therefore higher for loci with many alleles and for which $\sigma_{p}^{2}$ is small. A favorable aspect for studying the molecular diversity of populations is provided when microsatellite markers are used because a large number of alleles is generally detected. For example, the potential range of $h$ for a locus with three alleles, is 0 to 0.67 and for a locus with 10 alleles is 0 to 0.9 and consequently there is greater sensitivity in detecting diversity when microsatellite markers are used in comparison to other markers. This favorable aspect is also observed when populations are subdivided and total diversity is split into components between and within subpopulations, as proposed by Nei (1973).

In investigations involving several natural subpopulations belonging to a metapopulation, the use of microsatellite markers results in a considerably higher number of exclusive or private alleles, which is very important for estimating the degree of isolation of the subpopulations. However, when dealing with parameters such as the effective populations size $\left(N_{e}\right)$ that are used for measuring the drift of gene frequencies due to sampling occurred preceding generations it is questionable if microsatellite markers are adequate. In this case Vencovsky and Crossa (2003) have shown that Wright's $F$ statistics $\left(e . g . F_{S T}\right.$ and $\left.F_{I T}\right)$ are fundamental for estimating the effective populations size of samples. A random model is required because interpopulation diversity in a given generation is a consequence of drift alone, when microsatellite mutation rates are high a random model is no longer applicable and estimates will be biased.

\section{Acknowledgments}

The authors would like to thank Ricardo V. Cesar for his kind contribution in the proofreading of this review.

\section{References}

Amos W, Sawcer SJ, Feakes RW and Rubinsztein DC (1996) Microsatellites show mutational bias and heterozygote instability. Nature Genetics 13:390-391.

Arcot SS, Wang Z, Weber JL, Deininger L and Batzer MA (1995) Alu repeats: A source for the genesis of primate microsatellites. Genomics 29:136-144.

Avise J (1994) Molecular Markers, Natural History and Evolution. Chapman \& Hall, New York, 511 pp.

Bachtrog D, Weiss S, Zangerl B, Brem G and Schlötterer C (1999) Distribution of dinucleotide microsatellites in the Drosophila melanogaster genome. Molecular Biology and Evolution 16:602-610.

Bailey AD, Pavelitz T and Weiner AM (1998) The microsatellite sequence $(\mathrm{CT}) \mathrm{n} \times(\mathrm{GA}) \mathrm{n}$ promotes stable chromosomal integration of large tandem arrays of functional human U2 small nuclear RNA genes. Molecular and Cell Biology 18:22262271.

Balloux F and Goudet J (2002) Statistical properties of population differentiation estimators under stepwise mutation in a finite island model. Molecular Ecology 11:771-783.

Balloux F and Lugon-Moulin N (2002) The estimation of population differentiation with microsatellite markers. Molecular Ecology 11:155-165.

Balloux F, Lugon-Moulin N and Hausser J (2000) Estimating gene flow across hybrid zones: How reliable are microsatellites? Acta Theriologica 45:93-101.

Belkum A Van (1999) Short sequence repeats in microbial pathogenesis and evolution. Cellular and Molecular Life Sciences 56:729-734.

Bernardo R, Murigneux A and Karaman Z (1996) Marker-based estimates of identity by descent and alikeness in state among maize inbreds. Theoretical and Applied Genetics 93:262267.

Bichara M, Pinet I, Schumacher S and Fuchs R (2000) Mechanisms of dinucleotide repeat instability in Escherichia coli. Genetics 154:533-542.

Biggin MD and Tjian R (1988) Transcription factors that activate the ultrabithorax promoter in developmentally staged extracts. Cell 53:699-711.

Blouin MS (2003) DNA-based methods for pedigree reconstruction and kinship analysis in natural populations. Trends in Ecology and Evolution 18:503-511.

Bowers J, Boursiquot JM, This P, Chu K, Johansson H and Meredith C (1999) Historical genetics: The parentage of Chardonnay, Gamay, and other wine grapes of northeastern France. Science 285:1562-1565.

Bowen BW, Bass AL, Soares L and Toonen RJ (2005) Conservation implications of complex population structure: Lessons from the loggerhead turtle (Caretta caretta). Molecular Ecology 14:2389-2402.

Brondani RV, Brondani C, Tarchini R and Grattapaglia D (1998) Development, characterization and mapping of microsatellite markers in Eucalyptus grandis and E. urophylla. Theoretical and Applied Genetics 97:816-827.

Chakraborty R, Meagher TR and Smouse PE (1988) Parentage analysis with genetic-markers in natural-populations. 1 . The 
expected proportion of offspring with unambiguous paternity. Genetics 118:527-536.

Chakraborty R, Stivers DN, Su B, Zhong YX and Budowle B (1999) The utility of short tandem repeat loci beyond human identification: Implications for development of new DNA typing systems. Electrophoresis 20:1682-1696.

Ciampi AY (1999) Desenvolvimento e utilização de marcadores microsatélites, AFLP e seqüenciamento de cpDNA, no estudo da estrutura genética e parentesco em populações de copaíba (Copaifera langsdorffii) em matas de galeria no cerrado. PhD Thesis, Universidade Estadual Paulista 'Júlio de Mesquita Filho', Botucatu, 204 pp.

Cipriani G, Lot G, Huang WG, Matarazzo MT, Peterlunger E and Testolin R (1999) AC/GT and AG/CT microsatellite repeats in peach [Prunus persica (L) Batsch]: Isolation, characterisation and cross species amplification in Prunus. Theoretical and Applied Genetics 99:65-72.

Collevatti RG, Brondani RV and Grattapaglia D (1999) Development and characterization of microsatellite markers for genetic analysis of a Brazilian endangered tree species Caryocar brasiliense. Heredity 83:748-756.

Collevatti RG, Grattapaglia D and Hay JD (2001) Population genetic structure of the endangered tropical tree species Caryocar brasiliense, based on variability at microsatellite loci. Molecular Ecology 10:349-356.

Cooper G, Rubinsztein DC and Amos W (1998) Ascertainment bias cannot entirely account for human microsatellites being longer than their chimpanzee homologues. Human Molecular Genetics 7:1425-1429.

Cregan B, Mudge J, Fickus EW, Marek LF, Danesh D, Denny R, Shoemaker RC, Matthews BF, Jarvik T and Young ND (1999) Targeted isolation of simple sequence repeat markers through the use of bacterial artificial chromosomes. Theoretical and Applied Genetics 98:919-928.

Crollius HR, Jaillon O, Dasilva C, Ozouf Costaz C, Fizames C, Fischer C, Bouneau L, Billault A, Quetier F, Saurin W, Bernot A and Weissenbach J (2000) Characterization and repeat analysis of the compact genome of the freshwater pufferfish Tetraodon nigroviridis. Genome Research 10:939-949.

Crow J and Kimura M (1970) An Introduction to Population Genetics Theory. Burgess Publishing Company, Minneapolis, $591 \mathrm{pp}$.

Cummings CJ and Zoghbi HY (2000) Trinucleotide repeats: Mechanisms and pathophysiology. Annual Review of Genomics and Human Genetics 1:281-328.

Dayanandan S, Bawa KS and Kesseli R (1997) Conservation of microsatellites among tropical tree (Leguminosae). American Journal of Botany 84:1658-1663.

Di Rienzo A, Donnelly P, Toomajian C, Sisk B, Hill A, Petzl-Erle ML, Haines GH and Barch DH (1998) Heterogeneity of microsatellite mutations within and between loci, and implications for human demographic histories. Genetics 148:1269-1284.

Di Rienzo A, Peterson AC, Garza JC, Valdes AM, Slatkin M and Freimer NB (1994) Mutational processes of simple sequence repeat loci in human populations. Proceeding of National Academy of Sciences 91:3166-3170.

Donnelly MJ, Pinto J, Girod R, Besansky NJ and Lehmann T (2004) Revisiting the role of introgression vs. shared ancestral polymorphisms as key processes shaping genetic diver- sity in the recently separated sibling species of the Anopheles gambiae complex. Heredity 92:61-68.

Dow BD, Ashley MV and Howe HF (1995) Isolation and characterization of highly variable $(\mathrm{GA} / \mathrm{CT}) \mathrm{n}$ microsatellites in the bur oak, Quercus macrocarpa. Theoretical and Applied Genetics 91:137-141.

Dow BD and Ashley MV (1996). Microsatellite analysis of seed dispersal and sapling parentage in bur oak, Quercus macrocarpa. Molecular Ecology 5:615-627.

Ellegren H, Primmer CR and Sheldon B (1995) Microsatellite evolution: Directionality or bias in locus selection? Nature Genetics 11:60-62.

Estoup A, Cornuet JM, Rousset F and Guyomard R (1999) Juxtaposed microsatellite systems as diagnostic markers for admixture: Theoretical aspects. Molecular Biology and Evolution 16:898-908.

Estoup A, Jarne P and Cornuet JM (2002) Homoplasy and mutation model at microsatellite loci and their consequences for population genetics analysis. Molecular Ecology 11:15911604.

Everett CM and Wood NW (2004) Trinucleotide repeats and neurodegenerative disease. Brain 127:2385-2405.

Falconer DS (1960) Introduction to Quantitative Genetics. The Ronald Press, New York, 365 pp.

Field D and Wills C (1998) Long polymorphic microsatellites in simple organisms. Proceeding of the Royal Society of London, Series B: Biological Sciences 263:209-215.

Foster L and Trimarchi JM (1994) Adaptive reversion of a frameshift mutation in Escherichia coli by simple base deletions in homopolymeric runs. Science 265:407-409.

Gaggiotti OE, Lange O, Rassmann K and Gliddon C (1999) A comparison of two indirect methods for estimating average levels of gene flow using microsatellite data. Molecular Ecology 8:1513-1520.

Garner TWJ, Angelone S and Pearman B (2003) Genetic depletion in Swiss populations of Rana latastei: Conservation implications. Biological Conservation 114:371-376.

Gilmour DS, Thomas GH and Elgin SC (1989) Drosophila nuclear proteins bind to regions of alternating $\mathrm{C}$ and $\mathrm{T}$ residues in gene promoters. Science 245:1487-1490.

Goldstein DB and Schlotterer C (1999) Microsatellites: Evolution and Applications. Oxford University Press, New York, 343 pp.

Hancock JM (1999) Microsatellites and other simple sequences: Genomic context and mutational mechanisms. In: Goldstein DB and Schlötterer C (eds) Microsatellites: Evolution and Applications. Oxford University Press, New York, p 1-9.

Hedrick W (2001) Conservation genetics: Where are we now? Trends in Ecology and Evolution 16:629-636.

Hentschel CC (1982) Homocopolymer sequences in the spacer of a sea urchin histone gene repeat are sensitive to $\mathrm{S} 1$ nuclease. Nature 295:714-716.

Heywood VH and Iriondo JM (2003) Plant conservation: Old problems, new perspectives. Biological Conservation 113:321-335.

Hood DW, Deadman ME, Jennings M, Bisercic M, Fleischmann RD, Venter JC and Moxon ER (1996) DNA repeats identify novel virulence genes in Haemophilus influenzae. Proceedings of the National Academy of Sciences 93:11121-11125.

Hunter BR (1989) ASTA approach on minimum distance. In: Wilkinson D (ed) Proceedings Annual Corn and Sorghum 
Industry Research Conference, 44:193-195. American Seed Trade Association, Washington.

Isagi Y and Suhandono S (1997) PCR primers amplifying microsatellite loci of Quercus myrsinifolia Blume and their conservation between oak species. Molecular Ecology 6:897-899.

Jackson PJ, Walthers EA, Kalif AS, Richmond KL, Adair DM, Hill KK, Kuske CR, Andersen GL, Wilson KH, Hughjones ME and Keim P (1997) Characterization of the variable number tandem repeats in VITA from different Bacillus anthracis isolates. Applied Environment Microbiology 63:1400-1405.

Jarne P and Lagoda PJL (1996) Microsatellites, from molecules to populations and back. Trends in Ecology and Evolution 11:424-429.

Kalinowski ST (2002) How many alleles per locus should be used to estimate genetic distances? Heredity 88:62-65.

Kashi Y and Soller M (1999) Functional roles of microsatellites and minisatellites. In: Goldstein DB and Schlötterer C (eds) Microsatellites: Evolution and Applications. Oxford University Press, New York, pp 10-23.

Kempthorne O (1957) An Introduction to Genetic Statistics. Willey, New York, 545 pp.

Knapik EW, Goodman A, Ekker M, Chevrette M, Delgado J, Neuhauss S, Shimoda N, Driever W, Fishman MC and Jacob HJ (1998) A microsatellite genetic linkage map for zebrafish (Danio rerio). Nature Genetics 18:338-343.

Kolodner RD and Marsischky GT (1999) Eukaryotic DNA mismatch repair. Current Opinion Genetics 9:89-96.

Kruglyak S, Durret RT, Schug M and Aquadro CF (1998) Equilibrium distributions of microsatellite repeat length resulting from a balance between slippage events and point mutations. Proceeding of National Academy of Sciences 95:1077410778.

Lemes MR, Gribel R, Proctor J and Grattapaglia D (2003) Population genetic structure of mahogany (Swietenia macrophylla King, Meliaceae) across the Brazilian Amazon, based on variation at microsatellite loci: Implications for conservation. Molecular Ecology 12:2875-2883.

Levinson G and Gutman GA (1987) Slippedstrand mispairing: A major mechanism for DNA sequence evolution. Molecular and Biology Evolution 4:203-221.

Lin X, Kaul S, Rounsley S, Shea T, Benito MI, Town CD, Fujii CY, Mason T, Bowman CL, Barnstead M, Feldblyum TV, Buell CR, Ketchum KA, Lee J, Ronning CM, Koo HL, Moffat KS, Cronin LA, Shen M, Pai G, Van Aken S, Umayam L, Tallon LJ, Gill JE and Venter JC (1999) Sequence and analysis of chromosome 2 of the plant Arabidopsis thaliana. Nature 402:761-768.

Lowenhaupt K, Rich A and Pardue ML (1989) Nonrandom distribution of long mono and dinucleotide repeats in Drosophila chromosomes: Correlations with dosage compensation, heterochromatin, and recombination. Molecular and Cell Biology 9:1173-1182.

Lynch M and Ritland K (1999) Estimation of pairwise relatedness with molecular markers. Genetics 152:1753-1766.

Mahadevan M, Tsilfidis C, Sabourin L, Shutler G, Amemiya C, Jansen G, Neville C, Narang M, Barcelo J, O’Hoy K, Leblond S, Earle Macdonald J, De Jong J and Wieringa B (1992) Myotonic dystrophy mutation: An unstable CTG re- peat in the 38 untranslated region of the gene. Science 255:1253-1258.

Malécot G (1948) Les Mathematiques de L’Herédité. Masson, Paris, $63 \mathrm{pp}$.

Messier W, Li SH and Stewart CB (1996) The birth of microsatellites. Nature 381:483.

Métais I, Hamon B, Jalouzot R and Peltier D (2002) Structure and level of genetic diversity in various bean types evidenced with microsatellite markers isolated from a genomic enriched library. Theoretical and Applied Genetics 104:13461352.

Metzgar M, Bytof J and Wills C (2000) Selection against frameshift mutations limits microsatellite expansion in coding DNA. Genome Research 10:72-80.

Morgante M, Hanafey M and Powell W (2002) Microsatellites are preferentially associated with nonrepetitive DNA in plant genomes. Nature Genetics 30:194-200.

Morin PA, Mahboubi P, Wedel S and Rogers J (1998) Rapid screening and comparison of human microsatellite markers in baboons: Allele size is conserved, but allele number is not. Genomics 53:12-20.

Moxon R and Willis C (1999) DNA microsatellites: Agents of evolution? Scientific American 280:94-99.

Nadir E, Margalit H, Gallily T and Bensasson SA (1996) Microsatellite spreading in the human genome: Evolutionary mechanisms and structural implications. Proceedings of the National Academy of Sciences 93:6470-6475.

Nei, M (1973) Analysis of genetic diversity in subdivided populations. Proceedings of the National Academy of Sciences 70:3321-3323.

Nichols RA and Freeman KLN (2004) Using molecular markers with high mutation rates to obtain estimates of relative population size and to distinguish the effects of gene flow and mutation: A demonstration using data from endemic Mauritian skinks. Molecular Ecology 13:775-787.

Novick RR, Dick CW, Lemes MR, Navarro C, Caccone A and Bermingham E (2003) Genetic structure of Mesoamerican populations of big-leaf mahogany (Swietenia macrophylla) inferred from microsatellite analysis. Molecular Ecology 12:2885-2893.

O'Donnell KH, Chen CT and Wensink C (1994) Insulating DNA directs ubiquitous transcription of the Drosophila melanogaster alpha1 tubulin gene. Molecular and Cell Biology 14:6398-6408.

O'Donnell WT and Warren ST (2002) A decade of molecular studies of fragile X syndrome. Neurosciences 25:315-338.

Pardue ML, Lowenhaupt K, Rich A and Nordheim A (1987) $(\mathrm{dCdA}) \mathrm{n}(\mathrm{dGdT}) \mathrm{n}$ sequences have evolutionarily conserved chromosomal locations in Drosophila with implications for roles in chromosome structure and function. Embo Journal 6:1781-1789.

Petit RJ, Deguilloux MF, Chat J, Grivet D, Garnier-Géré P and Vendramin GG (2005) Standardizing for microsatellite length in comparisons of genetic diversity. Molecular Ecology 14:885-890.

Pinto LR, Vieira MLC, Souza Jr CL and Souza AP (2003a) Reciprocal recurrent selection effects on the genetic structure of tropical maize populations assessed at microsatellite loci. Genetics and Molecular Biology 26:355-364.

Pinto LR, Vieira, MLC, Souza Jr CL and Souza AP (2003b) Genetic-diversity assessed by microsatellites in tropical maize 
populations submitted to a high-intensity reciprocal recurrent selection. Euphytica 134:277-286.

Primmer CR and Merilä J (2000) A low rate of cross-species microsatellite amplification success in Ranid frogs. Conservation Genetics 3:445-449.

Pupko T and Graur D (1999) Evolution of microsatellites in the yeast Saccharomyces cerevisiae: Role of length and number of repeated units. Journal of Molecular Evolution 48:313316.

Queller DC and Goodnight KF (1989) Estimation of genetic relatedness using allozyme data. Evolution 43:258-275.

Ramsay L, Macaulay M, Cardle L, Morgante M, Ivanissevich SD, Maestri E, Powell W and Waugh R (1999) Intimate association of microsatellite repeats with retrotransposons and other dispersed repetitive elements in barley. The Plant Journal 17:415-425.

Ritland K (1996) Estimators for pairwise relatedness and individual inbreeding coefficients. Genetical Research 67:175-185.

Roa AC, Chavarriaga-Aguirre P, Duque MC, Maya MM, Bonierbale MW, Iglesias C and Tohme J (2000) Cross species amplification of cassava (Manihot esculenta) (Euphorbiaceae) microsatellites: Allelic polymorphism and degree of relationship American Journal of Botany 87:1647-1655.

Rose O and Falush D (1998) A threshold size for microsatellite expansion. Molecular Biology and Evolution 15:613-615.

Rosenberg SM, Longerich S, Gee P and Harris RS (1994) Adaptive mutation by deletions in small mononucleotide repeats. Science 265:405-407.

Rubinsztein DC, Amos W, Leggo J, Goodburn S, Jain S, Li SH, Margolis RL, Ross CA and Fergusonsmith MA (1995) Microsatellite evolution - Evidence for directionality and variation in rate between species. Nature Genetics 10:337343.

Saunders NJ, Peden JF, Hood DW and Moxon ER (1998) Simple sequence repeats in the Helicobacter pylori genome. Molecular Microbiology 27:1091-1098.

Schlötterer C (2000) Evolutionary dynamics of microsatellite DNA. Chromossoma 109:365-371.

Schlötterer C, Amos B and Tautz D (1991) Conservation of polymorphic simple sequence loci in cetacean species. Nature 354:63-65.

Schlotterer C and Harr B (2000) Drosophila virilis has long and highly polymorphic microsatellites. Molecular Biology and Evolution 17:1641-1646.

Schlötterer C and Tautz D (1992) Slippage synthesis of simple sequence DNA. Nucleic Acids Research 20:211-215.

Sia EA, Kokoska RJ, Dominska M, Greenwell P and Petes TD (1997) Microsatellite instability in yeast: Dependence on repeat unit size and DNA mismatch repair genes. Molecular and Cellular Biology 17:2851-2858.

Sia EA, Butler CA, Dominska M, Greenwell P, Fox TD, and Petes TD (2000). Analysis of microsatellite mutations in the mitochondrial DNA of Saccharomyces cerevisiae. Proceedings of the National Academy of Sciences 97:250-255.

Slatkin M (1995) A measure of population subdivision based on microsatellite allele frequencies. Genetics 139:457-462.

Song QJ, Fickus EW and Cregan PB (2002) Characterization of trinucleotide SSR motifs in wheat. Theoretical and Applied Genetics 104:286-293.
Souza E and Sorrells ME (1989) Pedigree analysis of northamerican oat cultivars released from 1951 to 1985 . Crop Science 29:595-601.

Stallings RL (1994) Distribution of trinucleotide microsatellites in different categories of mammalian genomic sequence: Implication for human genetic diseases. Genomics 21:116121.

Steinkellner H, Lexer C, Turetschek E and Gloss1 J (1997) Conservation of (GA)(n) microsatellite loci between Quercus species. Molecular Ecology 6:1189-1194.

Strand M, Prolla TA, Liskay RM and Petes TD (1993) Destabilization of tracts of simple repetitive DNA in yeast by mutations affecting DNA mismatch repair. Nature 365:274-276.

Streisinger G and Owen JE (1985) Mechanisms of spontaneous and induced frameshift mutation in bacteriophage T4. Genetics 109:633-659.

Thompson EA and Meagher TR (1998) Genetic linkage in the estimation of pairwise relationship. Theoretical and Applied Genetics 97:857-864.

Tóth G, Gáspari Z and Jurka J (2000) Microsatellites in different eukaryotic genomes: Survey and analysis. Genome Research 10:967-981.

Treco D and Arnheim N (1986) The evolutionarily conserved repetitive sequence d(TG.AC)n promotes reciprocal exchange and generates unusual recombinant tetrads during yeast meiosis. Molecular and Cell Biology 6:3934-3947.

Vencovsky R and Crossa J (2003) Measurements of representativeness used in genetic resources conservation and plant breeding. Crop Science 43:1912-1921.

Wahls W, Wallace LJ and Moore D (1990) The Z-DNA motif d(TG)30 promotes reception of information during gene conversion events while stimulating homologous recombination in human cells in culture. Molecular and Cell Biology 10:785-793.

Weir BS (1996) Genetic Data Analysis II. Sinauer, Sunderland, $445 \mathrm{pp}$.

White G and Powell W (1997) Isolation and characterization of microsatellite loci in Swietenia humilis (Meliaceae): An endangered tropical hardwood species. Molecular Ecology 6:851-860.

Wilder J and Hollocher H (2001) Mobile elements and the genesis of microsatellites in dipterans. Molecular Biology and Evolution 18:384-392.

Williamson JE, Huebinger RM, Sommer JA, Louis EE and Barber RC (2002) Development and cross species amplification of 18 microsatellite markers in the Sumatran tiger (Panthera tigris sumatrae). Molecular Ecology Notes 2:110-112.

Wright S (1922) Coefficients of inbreeding and relationship. American Naturalist 56:330-338.

Wright S (1931) Evolution in Mendelian populations. Genetics 16:97-159.

Wright S (1932) The roles of mutation, inbreeding, crossbreeding, and selection in evolution. Proceedings of the Sixth International Congress on Genetics 1:356-366.

Wright S (1951) The genetical structure of populations. Annual Eugenics 15:323-354.

Wright JM and Bentzen P (1994) Microsatellites: Genetic markers of the future. Reviews in Fish Biology and Fisheries 4:384-388. 
Zhu Y, Strassmann JE and Queller DC (2000) Insertions, substitutions, and the origin of microsatellites. Genetics Research 76:227-236.

Zucchi MI, Brondani RV, Pinheiro JB, Brondani C and Vencovsky R (2002) Transferability of microsatellite markers from Eucalyptus spp. to Eugenia dysenterica (Myrtaceae family). Molecular Ecology Notes 2:512-514.

Zucchi MI, Brondani RV, Pinheiro, JB, Coelho ASG, Chaves LJ and Vencovsky R (2003) Genetic structure and gene flow in Eugenia dysenterica DC in the Brazilian cerrado utilizing SSR markers. Genetics and Molecular Biology 26:449-458.

Associate Editor: Everaldo Gonçalves de Barros 